\title{
Steering the propagation direction of a non-linear acoustic wave in a solid material
}

\author{
Hector Hernandez Delgadillo, ${ }^{\mathrm{a}, \mathrm{b},}$, Richard Loendersloot $^{\mathrm{a}}$, Doekle Yntema ${ }^{\mathrm{b}}$, Tiedo Tinga ${ }^{\mathrm{a}}$, \\ Remko Akkerman ${ }^{\mathrm{a}}$ \\ ${ }^{\text {a }}$ Faculty of Engineering Technology (ET), University of Twente, Enschede, the Netherlands \\ ${ }^{\mathrm{b}}$ Smart Water Grids Theme, Wetsus European Centre of Excellence for Sustainable Water Technology, Leeuwarden, the Netherlands
}

\section{A R T I C L E I N F O}

\section{Keywords:}

Non-collinear wave mixing

Steering

Generated wave

Interaction angle

\begin{abstract}
A B S T R A C T
In this research non-collinear wave mixing is used as a non-destructive testing method where the amplitude of the scattering wave contains information on the condition of a material. The practical implementation of noncollinear wave mixing as a non-destructive testing technique is limited by many factors such as the geometry and shape of the structure, the accessibility to the specimen's surfaces and the ultrasonic sensors available to perform measurements. A novel approach to steer the propagation direction of a generated wave from the mixing of two incident acoustic waves is proposed. The angle of the scattering wave is controlled by the frequencies of the two interaction waves, rather than by the angle between these waves. The scattering amplitude was analytically solved for the longitudinal plus shear interaction process. The analytical solution was validated with experiments. The model qualitatively agrees with the experiments. Furthermore, the possibility to use a wider range of excitation frequencies of the incident waves was found. This is a great advantage in applications where the space and access to the specimen under test is limited.
\end{abstract}

\section{Introduction}

In recent years, the generation of an acoustic wave from the mixing of two incident waves has been of interest for researchers because of its great advantages. Applications such as detection of plasticity in metals, detection of micro-cracks, fatigue and detection of physical ageing in plastics make it very attractive [1-7]. Furthermore, the detection of imperfect interfaces with the wave mixing technique has been a subject of research as well [8-12]. For instance, the measured amplitude of the generated wave was found to be directly related to the acoustic parameter $\beta$. Direct correlation was found between this parameter and damage in solids such as low adhesive joint quality, weathering damage in limestone blocks and plastic deformation in aluminium [3,13-15]. The measured amplitude of the generated wave has a relatively high sensitivity to any of the changes in the conditions mentioned above compared to linear ultrasonics. In recent work, Demčenko et al. [5] demonstrated that with two-sided non-collinear wave mixing configuration it is possible to detect ageing in grey polyvinyl chloride (PVC) with good sensitivity compared to linear ultrasonics in the longitudinal wave mode [5].

The scattering field of a wave generated from the local resonance of two incident waves in a solid medium was derived in 1968 from the linear theory of elasticity and with the time-dependent perturbation theory [16-19]. In a more recent research, Korneev et al. [20] presented a corrected version of the derivation done in 1968, as well as the solution of an amplitude coefficient for the ten possible interaction processes. The solutions of these are aimed to be used to find the optimal testing parameters [20].

In the available literature, the propagation direction is calculated from the input frequencies of the incident waves. In none of the work found, the direction of the generated wave is steered. This means that no experimental set-up has been designed such that it has the accuracy to change the angles and distances between sensors and the testing material. The possibility to mechanically steer the generated wave becomes challenging in applications limited by the space, weight and energy available. When the access to only one surface is possible, the complexity of a testing system further increases. It is commonly found that for laboratory set-ups, the access to more than one surface is attainable. However, positioning of the sensors in many cases for real testing of structures has limitations. For instance, the inspection of PVC pipes requires access to only the internal or external surface. Thus, in order to upscale a testing configuration towards non-destructive

\footnotetext{
* Corresponding author.

E-mail address: h.hernandezdelgadillo@outlook.com (H. Hernandez Delgadillo).
} 
evaluation (NDE) purpose, the settings have to be optimized.

The objective of this research is the optimization of a wave mixing configuration. This is done by demonstrating from experiments the possibility to steer the propagation direction of a wave generated from the mix of two incident waves by changing the incident wave's excitation frequencies only. The latter is achieved while keeping the two incident angles constant. This considerably simplifies the testing conditions, as the two sensors need to be positioned just once. Thereafter, solely the receiver has to be adjusted according to the propagation direction of the generated wave. Furthermore, by having a constant interaction angle and a variable excitation frequency, the optimal settings can be directly adjusted by changing the pump wave's frequencies when the receiver's location cannot be adjusted.

The outline of this paper is as follows. In Section 2, the wave mixing theory will be shortly discussed. In Section 3, the methodology is explained. First, in the analytical part, the assumptions to the solution of the equations shown in Section 2 are explained. In the second part of Section 3, the experimental campaign used to validate the analytical solution is described in detail. Section 4 then presents and discusses all the results of both the analytical and experimental work, and finally Section 5 forwards the main conclusions.

The main contributions of this research are: (i) providing the solution of the scattering amplitude and the interaction volume for this interaction process as a function of the second pump wave frequency at a constant interaction angle $\alpha$; (ii) presenting the idea to steer the direction of the generated wave by only changing the frequencies of the incident waves, based on this solution. Thus, significantly reducing the complexity of a test set-up and still allowing optimization of the generated wave.

\section{Wave mixing theory}

The local resonance of two incident acoustic waves generates a third acoustic wave which propagates at an angle $\psi$ with respect to one of the incident waves. In Fig. 1 this phenomenon is depicted where the incident wave vectors are $\mathbf{k}_{1}$ and $\mathbf{k}_{2}$ and $\mathbf{k}_{g}$ is the generated wave vector.

The interaction of these two waves is defined by the cosine law

$\left(\mathbf{k}_{1}\right)^{2}+\left(\mathbf{k}_{2}\right)^{2} \pm 2 \overline{\mathbf{k}}_{1} \overline{\mathbf{k}}_{2} \cos \alpha=\left(\mathbf{k}_{g}\right)^{2}$

where $\alpha$ is the interaction angle between $k_{1}$ and $k_{2}$ and the magnitude of the wave vectors is

$\overline{\mathbf{k}}_{n}=\frac{\omega_{n}}{\overline{\mathbf{v}}_{n}}$,

where $\omega_{n}$ is the frequency of the incident waves (1 and 2) and the generated wave $(g), \overline{\mathbf{v}}_{n}$ is either the longitudinal or shear speed of sound in the material. The generated wave vector propagates under an angle with respect to $\mathbf{k}_{1}$ equal to

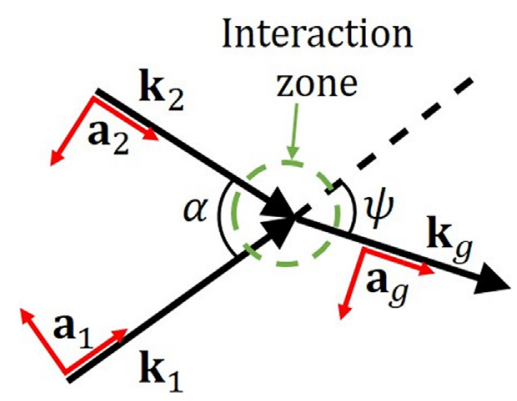

Fig. 1. Wave $\mathbf{k}_{\mathbf{g}}$ generated from the local resonance of two incident acoustic waves $\left(\mathbf{k}_{\mathbf{1}}\right.$ and $\mathbf{k}_{\mathbf{2}}$ ). The symbol $\mathbf{k}_{\mathbf{i}}$ indicates the wave vectors and $\mathbf{a}_{\mathbf{i}}$ the polarization of the waves. $\psi=\tan ^{-1}\left(\frac{ \pm \frac{v_{1}}{v_{2}} d \sin \alpha}{1 \pm \frac{v_{1}}{v_{2}} d \cos \alpha}\right)$

where $d=\omega_{2} / \omega_{1}$. This is depicted in Fig. 1 . The resonant conditions at which this process occurs are

$\mathbf{k}_{g}=\mathbf{k}_{1} \pm \mathbf{k}_{2}$

$\omega_{g}=\omega_{1} \pm \omega_{2}$

The local resonance of two incident waves cannot be described with the linear elastic theory if the equation of motion is linear due to its nonlinear nature $[18,20]$. The equation of motion in the Cartesian form is

$\rho \frac{\partial^{2} \mathbf{u}}{\partial t^{2}}-\mu \nabla^{2} \mathbf{u}-(\lambda+\mu) \nabla(\nabla \cdot \mathbf{u})=\mathbf{F}$,

where $\rho$ is the density of the material, $\mu$ and $\lambda$ are the Lamé parameters, $\mathbf{F}$ is an external force, $\mathbf{u}$ the particle displacement vector. In order to have a non-linear equation of motion which considers the interaction between two plane waves, cubic terms are included in the particle displacements $[18,20]$. The sum of the two incident waves is assumed to be

$\mathbf{u}_{0}=A_{1} \cos \left(\omega_{1} t-\left(\mathbf{k}_{1} \cdot \mathbf{r}\right)\right) \mathbf{a}_{1}+A_{2} \cos \left(\omega_{2} t-\left(\mathbf{k}_{2} \cdot \mathbf{r}\right)\right) \mathbf{a}_{2}$,

where $\mathbf{r}$ is the vector from the center of interaction to the observation point (position of receiver) and $\mathbf{a}_{1}$ and $\mathbf{a}_{2}$ are the polarization vectors of the incident waves. The polarization vector is parallel to the propagation direction for longitudinal waves (see Fig. 1), and perpendicular to the propagation direction for shear waves as shown in Fig. 1. The following is obtained from substituting Eq. (7) in Eq. (6)

$\mathbf{p}(\mathbf{r}, t)$

$$
\begin{aligned}
= & -A_{1} A_{2}\left(\mathbf{I}^{+} \sin \left[\left(\omega_{1}+\omega_{2}\right) t-\left(\mathbf{k}_{1}+\mathbf{k}_{2}\right) \mathbf{r}\right]+\mathbf{I}^{-} \sin \left[\left(\omega_{1}-\omega_{2}\right) t\right.\right. \\
& \left.\left.-\left(\mathbf{k}_{1}-\mathbf{k}_{2}\right) \mathbf{r}\right]\right),
\end{aligned}
$$

where $A_{1}$ and $A_{2}$ are the amplitudes of the incident waves. The \pm refers to the interaction process and it can be either the sum or the difference. In Eq. (8), the $\mathbf{p}$ vector is a component of the force $\mathbf{F}$ that contains the interactions of the acoustic waves $[18,20]$. This vector is a time dependent function and a function of the observation point (measurement location). The vector $\mathbf{I}^{ \pm}$is defined as

$\mathbf{I}^{ \pm}$

$$
\begin{aligned}
= & \frac{1}{2} C_{1}\left[\left(\mathbf{a}_{1} \cdot \mathbf{a}_{2}\right)\left(k_{2}^{2} \mathbf{k}_{1} \pm k_{1}^{2} \mathbf{k}_{2}\right)+\left(\mathbf{a}_{2} \cdot \mathbf{k}_{1}\right) k_{2}\left(k_{2} \pm 2 k_{1} \cos \alpha\right) \mathbf{a}_{1}+\left(\mathbf{a}_{1} \cdot \mathbf{k}_{2}\right) k_{1}\right. \\
& \left.\left(2 k_{2} \cos \alpha \pm k_{1}\right) \mathbf{a}_{2}\right]+\frac{1}{2} C_{2} k_{1} k_{2} \cos \alpha \\
& {\left[\left(\mathbf{a}_{1} \cdot \mathbf{a}_{2}\right)\left(\mathbf{k}_{2} \pm \mathbf{k}_{1}\right)+\left(\mathbf{a}_{2} \cdot \mathbf{k}_{2}\right) \mathbf{a}_{1} \pm\left(\mathbf{a}_{1} \cdot \mathbf{k}_{1}\right) \mathbf{a}_{1}\right]+\frac{1}{2} C_{3} } \\
& {\left[\left(\mathbf{a}_{1} \cdot \mathbf{k}_{2}\right)\left(\left(\mathbf{a}_{2} \cdot \mathbf{k}_{2}\right) \pm\left(\mathbf{a}_{2} \cdot \mathbf{k}_{1}\right)\right) k_{1}+\left(\mathbf{a}_{2} \cdot \mathbf{k}_{1}\right)\left(\left(\mathbf{a}_{1} \cdot \mathbf{k}_{2}\right) \pm\left(\mathbf{a}_{1} \cdot \mathbf{k}_{1}\right)\right) k_{2}\right]+\frac{1}{2} C_{4} } \\
& \left(\mathbf{a}_{2} \cdot \mathbf{k}_{2}\right)\left[\left(\mathbf{a}_{1} \cdot \mathbf{k}_{2}\right) \mathbf{k}_{2} \pm\left(\mathbf{a}_{1} \cdot \mathbf{k}_{1}\right) \mathbf{k}_{1}\right]+\frac{1}{2} C_{5}\left[\left(\mathbf{a}_{1} \cdot \mathbf{k}_{1}\right) \mathbf{k}_{2}^{2} \mathbf{a}_{2} \pm\left(\mathbf{a}_{2} \cdot \mathbf{k}_{2}\right) k_{1}^{2} \mathbf{a}_{1}\right],
\end{aligned}
$$

where $C_{1}$ to $C_{5}$ are functions of the third order elastic constant (TOEC) and the Lamé parameters $[18,20]$. In the vector $\mathbf{I}^{ \pm}$all the possible interactions are included. Thus, when selecting a specific interaction process, and depending on the type of wave (longitudinal or shear), the dot products within the vector will take a value of either one or zero. It is key to understand that the vector $\mathbf{I}^{ \pm}$contains the information of the material properties that change due to damage, which cannot be detected by linear ultrasonics. For example, the information contained in the vector $\mathbf{I}^{ \pm}$for the horizontal shear plus horizontal shear interaction process is captured by $C_{1}$ and $C_{2}$ only. The constants are defined as follow 

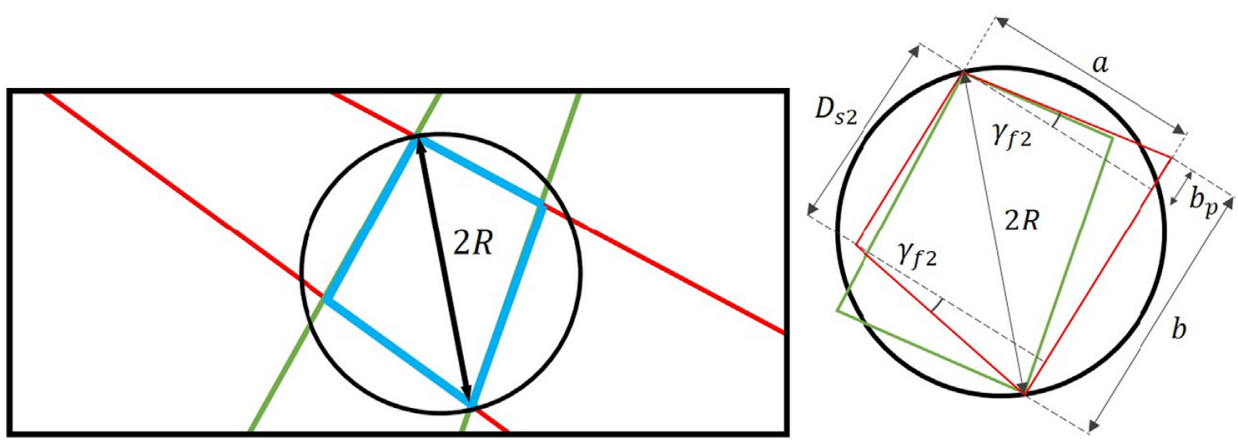

Fig. 2. Beam pattern of refracted waves inside the material showing how the beams of the refracted waves fit in the interaction zone. Trapezoid in red is the beam path of the refracted shear wave, the trapezoid in green is the beam path of the refracted longitudinal. (For interpretation of the references to color in this figure legend, the reader is referred to the web version of this article.)

$$
\begin{aligned}
& C_{1}=\mu+\frac{n}{4}, \quad C_{2}=\lambda+\mu+m-\frac{n}{4}, \quad C_{3}=m-\frac{n}{4}, \\
& C_{4}=2 l-m+\frac{n}{2}, \quad C_{5}=\lambda+m-\frac{n}{2},
\end{aligned}
$$

where $l, m$ and $n$ are the third order elastic constants. The complete derivation of the scattering field can be found in [18,20], and yields

$\mathbf{u}(\mathbf{r}, t)=\frac{A_{1} A_{2}}{4 \pi r \rho} \sum_{\xi=+,-}\left(\frac{\left(\mathbf{I}^{\xi} \cdot \hat{\mathbf{r}}\right) \hat{\mathbf{r}}}{v_{L}^{2}} V_{L}^{\xi}+\frac{\mathbf{I}^{\xi}-\left(\mathbf{I}^{\xi} \cdot \hat{\mathbf{r}}\right)}{v_{s}^{2}} V_{s}^{\xi}\right)$,

where $\hat{\mathbf{r}}$ is the unit vector of the observation point (measurement point), $v_{L}$ and $v_{s}$ are the longitudinal and shear speed of sound respectively and $V_{L}$ and $V_{s}$ are the interaction volumes if the generated wave is longitudinal or shear respectively. This solution entails all the possible interaction processes as it contains the vector $\mathbf{I}^{ \pm}$.

\section{Methodology}

In order to demonstrate that the generated wave can be steered other than by mechanically adjusting the interaction angles, the amplitude component from Eq. (11) was analytically solved for the longitudinal + shear (SV) interaction process. Furthermore, a set of experiments were performed in order to validate the solution for the amplitude of the generated wave as a function of the excitation frequencies.

\subsection{Analytical solution}

The scattering field from Eq. (11) for the longitudinal plus shear interaction process that generates a longitudinal wave is

$\mathbf{u}(\mathbf{r}, t)=\frac{A_{1} A_{2}}{4 \pi r \rho} \frac{\left(\mathbf{I}^{+} \cdot \hat{\mathbf{r}}\right) \hat{\mathbf{r}}}{v_{L}^{2}} V_{L}^{+}$.

The zone where the two incident beams interact is dependent on the wave length, speed of sound, amplitude and propagation direction of both beams (see Fig. 3). This zone is called the interaction volume. The given Equation is from [18]. The interaction volume for a generated longitudinal wave is defined as

$V_{L}^{+}=\int \sin \left(\left(\omega_{1}+\omega_{2}\right)\left(\frac{\mathrm{r}}{v_{g}}-t\right)-\left(\mathbf{k}_{1}+\mathbf{k}_{2}-\frac{\omega_{1}+\omega_{2}}{v_{g}} \hat{\mathbf{r}}\right) r^{\prime}\right) d V$.

Furthermore, the vector $\mathbf{I}^{ \pm}$for this interaction process is reduced to

$\mathbf{I}_{l+s}$

$$
\begin{aligned}
= & \frac{1}{2} C_{1}\left[\left(\mathbf{a}_{1} \cdot \mathbf{a}_{2}\right)\left(k_{2}^{2} \mathbf{k}_{1}+k_{1}^{2} \mathbf{k}_{2}\right)+\left(\mathbf{a}_{2} \cdot \mathbf{k}_{1}\right) k_{2}\left(k_{2}+2 k_{1} \cos \alpha\right) \mathbf{a}_{1}+\left(\mathbf{a}_{1} \cdot \mathbf{k}_{2}\right) k_{1}\right. \\
& \left.\left(2 k_{2} \cos \alpha+k_{1}\right) \mathbf{a}_{2}\right]+\frac{1}{2} C_{2} k_{1} k_{2} \cos \alpha\left[\left(\mathbf{a}_{1} \cdot \mathbf{a}_{2}\right)\left(\mathbf{k}_{2}+\mathbf{k}_{1}\right)+1\right]+\frac{1}{2} C_{3} \\
& {\left[\left(\mathbf{a}_{1} \cdot \mathbf{k}_{2}\right)\left(\left(\mathbf{a}_{2} \cdot \mathbf{k}_{1}\right)\right) k_{1}+\left(\mathbf{a}_{2} \cdot \mathbf{k}_{1}\right)\left(\left(\mathbf{a}_{1} \cdot \mathbf{k}_{2}\right)+1\right) k_{2}\right]+\frac{1}{2} C_{5} . }
\end{aligned}
$$

In this case only the $C_{4}$ component is zero. The general solution of the scattering amplitude depends on the integral of the interaction volume as shown in Eq. (13). If the volume of interaction is assumed to be a sphere, the amplitude of the scattering wave is

$\mathrm{A}=\frac{R^{3}}{3} \frac{\left(\mathbf{I}_{l+s}^{+} \cdot \hat{\mathbf{r}}\right)}{v_{l}^{2} \rho} \frac{A_{1} A_{2}}{r}$

where $R$ is the radius of the sphere. The radius is calculated by taking into account the diameter of the transducers and their beam divergence. Thus, the radius of the sphere is proposed to be defined as

$R=0.5 \sqrt{a^{2}+\left(D_{s 2}+\left(a \tan \gamma_{s 2}\right)\right)^{2}}$,

where $D_{s 2}$ is the diameter of the piezoelectric element of sensor two; $a$ is the distance that fits the width of beam path one to the length of beam path two; $b_{p}$ is the relative distance with respect to $a$ as a function of the beam divergence $\gamma_{s 2}$ of beam two. The beam divergence is calculated from the $6 \mathrm{~dB}$ decrease from the central beam path. Thus, the change in $f_{1}$ and $f_{2}$ are considered in terms $a$ and $\gamma_{s 2}$ respectively in Eq. (16). The interaction of the beam patterns is shown in Fig. 2. The beam paths are approximated as trapezoids. The proposed calculation of the interaction volume as a function of the pump wave frequencies is not available in the literature, and can thus be considered as a contribution of this research.

Values for the constants in Eq. (10), in this case for PVC are obtained from the literature [20]: $\lambda=3.64 \mathrm{GPa}, \mu=1.83 \mathrm{GPa}$, $l=-33.43 \mathrm{GPa}, m=-20.88 \mathrm{GPa}, n=-15.86 \mathrm{GPa}$ and $\rho=1350 \mathrm{~kg} / \mathrm{m}^{3}$.

\subsection{Experimental set-up}

The interaction process is $L_{1}+S_{2}=L$. The incident angle of the pump wave one $f_{1}$ was selected such that the refracted longitudinal wave is below the first critical angle. The amplitude of the refracted shear wave component before the first critical angle is much smaller than to the amplitude of the longitudinal wave (see Fig. 3b). The incident angle of the pump wave two $f_{2}$ was selected above the first critical angle (see Fig. 3a). The maximum amplitude of a shear wave component is achieved with an incident angle of approximately $50^{\circ}$ (see Fig. 3 b). Fig. 3 thus depicts the possible incident angles that will generate the refracted longitudinal and shear waves inside the PVC material. For these experiments, the refracted longitudinal angle is $\theta_{r l}=67^{\circ}$ and the refracted shear angle is $\theta_{r s}=31^{\circ}$. The interaction angle is $\alpha=98^{\circ}$.

A sinusoidal input voltage with 30 cycles (lowest frequency) and 40 cycles (highest frequency) were used for the pump waves. Despite that the bursts do not completely fit at the same time in the interaction zone, increasing the number of cycles improves the resolution of the generated wave in the frequency domain. No further interaction occurs after the waves travel beyond the interaction zone. A longer interaction is found not to be necessary. The excitation frequency of the first incident wave was kept constant while the excitation frequency of the second incident wave was gradually increased as shown in Table 1 .

Three ultrasonic transducers were placed according to the selected incident angles. The set-up is shown in Fig. 4, where the dimensions of the PVC samples are $l=150 \mathrm{~mm}, w=35 \mathrm{~mm}$ and thickness $t=15 \mathrm{~mm}$. 


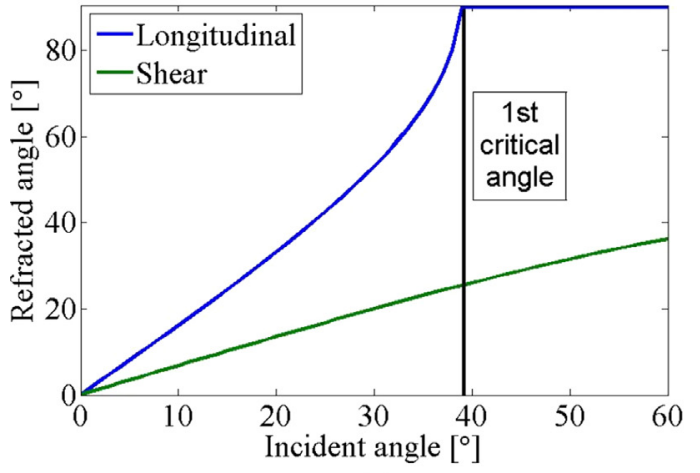

(a)

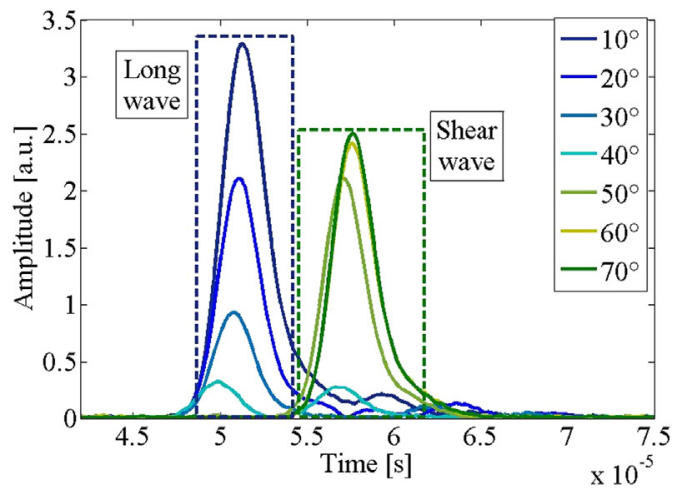

(b)

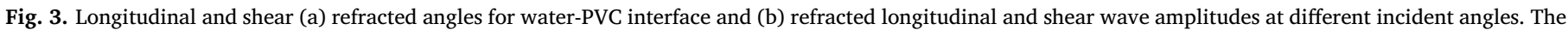
envelope of the measured signals is shown only.

Table 1

Input frequencies, frequency ratio $d=\omega_{2} / \omega_{1}$ and generated frequency for the interaction process.

\begin{tabular}{cccc}
\hline$d$ & $f_{1}(\mathrm{kHz})$ & $f_{2}(\mathrm{kHz})$ & $f_{g}(\mathrm{kHz})$ \\
\hline 1.3 & 650 & 850 & 1500 \\
1.384 & 650 & 900 & 1550 \\
1.461 & 650 & 950 & 1600 \\
1.538 & 650 & 1000 & 1650 \\
1.615 & 650 & 1050 & 1700 \\
1.692 & 650 & 1100 & 1750 \\
1.769 & 650 & 1150 & 1800 \\
1.846 & 650 & 1200 & 1850 \\
1.923 & 650 & 1250 & 1900 \\
2 & 650 & 1300 & 1950 \\
2.076 & 650 & 1350 & 2000 \\
\hline
\end{tabular}

The sensors used were two flat $1 \mathrm{MHz}$ central frequency transducers and the receiver used was a flat broad-band $2.25 \mathrm{MHz}$ central frequency transducer. The central frequency of these transducers is standard and is close to the frequencies shown in Table 1.

The interaction depth is calculated based on the initial positioning of the sensors. The initial position of the sensors are taken from literature where a longitudinal plus shear wave interaction was investigated in a PVC specimen [5]. The travel path of each wave is calculated as

$l_{\text {path }}=\frac{x_{m} \sin \left(90-\theta_{r s}\right)}{\sin \alpha}$,

$s_{\text {path }}=\frac{x_{m} \sin \left(90-\theta_{r l}\right)}{\sin \alpha}$,

where $x_{m}$ is the distance between the two beams refract in the material.

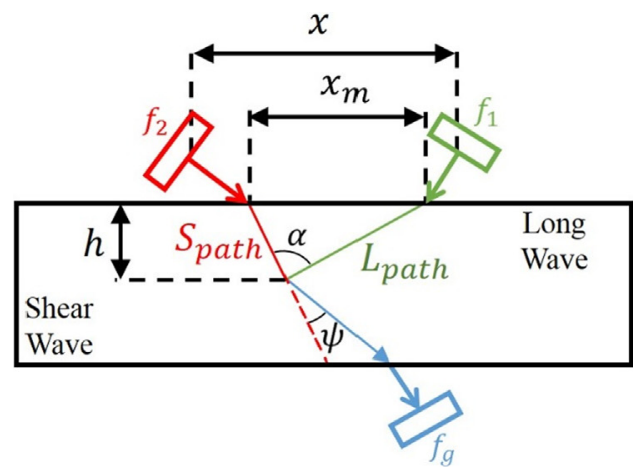

The interaction depth of these acoustic waves is calculated as

$h=l_{\text {path }} \cos \theta_{r l}=s_{\text {path }} \cos \theta_{r s}$.

The set-up including the electronic equipment is shown in Fig. 5. Before amplification, the voltage of the signal generator is $80 \mathrm{mV}$. The peak to peak voltage send to the transducers is $90 \mathrm{~V}$. The signal generator has two independent channels. These are synchronized by a trigger. The details on the manufacturers of the equipment used for this research are shown in Table 2.

The amplitude of the generated wave was recorded with a 15 bit resolution and $125 \mathrm{MS} / \mathrm{s}$ acquisition rate. During the experiments, a time-delay was applied to the second incident wave in order to compensate for the time-of-flight change due to the change in frequency due to the material dispersion [5]. The time-delay was adjusted until the maximum amplitude of the generated wave was found. From each experiment, the maximum peak of generated wave component in the frequency spectrum was extracted for 32 signals, averaged and then the standard deviation was calculated. The experimental set-up was disassembled and assembled for a second set of experiments.

The complete time-domain signals were transferred to the frequency domain as shown in Fig. 6. Three components are identified and the maximum amplitude of the generated wave frequency component is extracted as shown in Fig. 6.

The amplitude extracted from the frequency spectrum of the generated wave was corrected with the transfer function of the transducers. The data sheet from the transducer's manufacturer was used to derive a correction function. In this manner, the energy of the acoustic wave was compensated as the sensors were excited at frequencies other than the central frequency. Then the amplitude in the frequency spectrum was compensated as

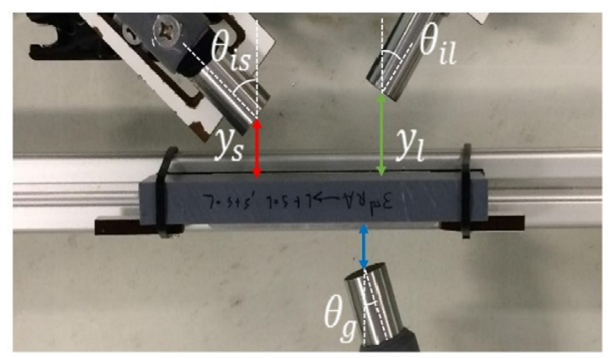

Fig. 4. Sensor positioning for longitudinal + shear interaction process. Interaction depth and the travel path of each wave component is depicted. 


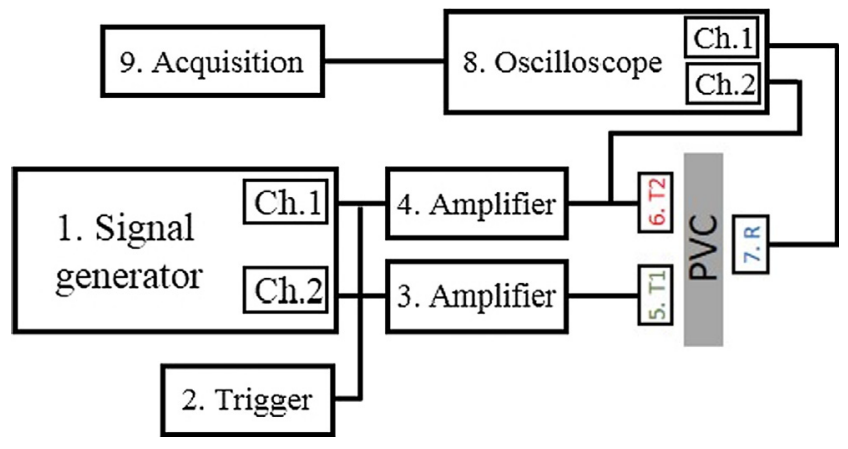

Fig. 5. Set-up including the electronic equipment.

Table 2

Set-up components.

\begin{tabular}{lll}
$\begin{array}{l}\text { 1. Signal generator } \\
\text { Keysight 33512B }\end{array}$ & 2. Trigger & 3. Amplifier \\
4. Amplifier & Aim-TTi TG 2000 & Tomco BTM00250 \\
Tomco BTM00250 & 5. Transmitter 1 & 6. Transmitter 2 \\
7. Receiver & Sofranel IBHG014 & Olympus I4-0110 \\
Olympus I4-0210 & 8. Oscilloscope & 9. Acquisition \\
& Picoscope 5442B & Computer \\
\hline
\end{tabular}

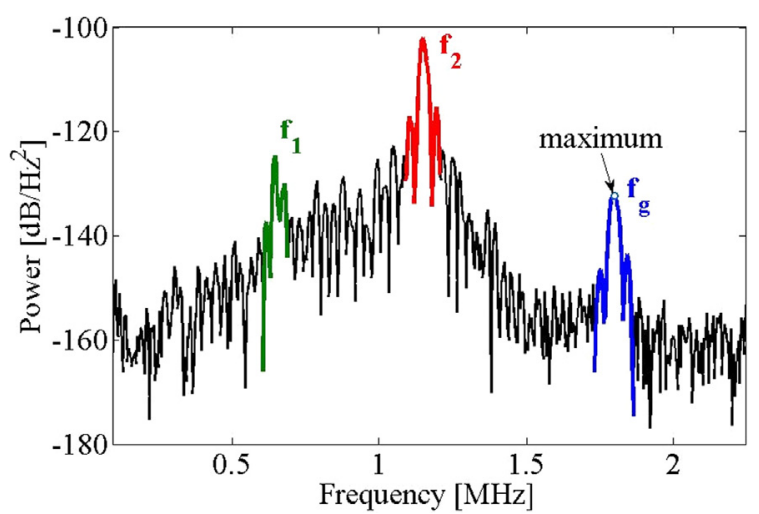

Fig. 6. Frequency spectrum of a typical signal from the wave mixing testing. The circle in blue is the maximum amplitude of the generated wave frequency component.

$A=\frac{A_{m}}{S_{T 2} S_{R}}$,

where $A_{m}$ is the amplitude of the generated wave in the frequency spectrum and $S_{T 2}, S_{R}$ are the transfer functions of the transmitter two and the receiver respectively. The propagation path of the generated wave is a function of the pump wave frequencies. It is assumed that the travel path change is small. Thus, the attenuation due to travel path change is neglected.

\section{Results and discussion}

\subsection{Analytical results}

The solution of Eq. (15) is calculated as a function of the frequency of pump wave two $\left(f_{2}\right)$ while frequency $f_{1}$ is left constant. This is done for many different constant values of $f_{1}$ pump frequencies, ranging from $300 \mathrm{kHz}$ to $1.5 \mathrm{MHz}$, with the correspondent interaction angle $\alpha$ for each $f_{1}$. The calculated propagation angle $\psi$ as a function of frequency $f_{2}$ and $\alpha$ is shown in Fig. 7a to Fig. 7f. Similarly, in Fig. 7a-f the solution to Eq. (15) is shown in the colour map for the different frequencies $f_{1}$ as a function of interaction angle $\alpha$ and the excitation frequency $f_{2}$. The amplitude of the generated wave is in arbitrary units, being dark blue low amplitude (0) and yellow for high amplitude (6).

A wide range of propagation directions can be seen in Fig. 7. For instance, $\psi$ linearly increases as $\alpha$ increases and vice versa. The propagation angle linearly increases with an increase in frequency $f_{2}$, however with lower rate. Furthermore, if the incident wave frequency $f_{1}$ is small, the range of propagation directions decreases (see Fig. 7a) compared to a higher $f_{1}$ frequencies. The increase in propagation is depicted in Fig. $7 \mathrm{a}$ to $7 \mathrm{f}$ on the left side of each figure. If $f_{1}$ increases, the propagation directions for $f_{2}<1 \mathrm{MHz}$ frequencies becomes available. Thus, the range of available propagation directions increases (see Fig. 7f). The information in Fig. 7 can be used to estimate the adequate pump frequencies for an experimental set-up based on the possible interaction angles that can be feasible to achieve. Once an interaction angle is fixed, the propagation direction can be modified by one of the two pump wave frequencies. This allows to have one single experimental set-up for the pump waves and only change the position of the receiver. This in turns reduces the complexity of an experimental setup.

In Fig. 7a-f, two main regions can be seen. The first has its maximum amplitude at $\alpha=45^{\circ}$ and the second has its maximum amplitude at $\alpha=135^{\circ}$. The region with the highest amplitude is the interaction angle $\alpha=45^{\circ}$. For both regions the amplitude of the generated wave increases with an increase in pump frequency $f_{2}$. Additionally, in the region around interaction angle $\alpha=100^{\circ}$, the amplitude for all the range of pump frequencies $f_{2}$ is the lowest. For the case of pump frequency $f_{1}=300 \mathrm{kHz}$ and at pump frequencies $f_{2}<1 \mathrm{MHz}$, the amplitude of the generated wave is the lowest for all interaction angles (see Fig. 7a). As the pump frequency $f_{1}$ is increased, the amplitude decreases around the interaction angle $\alpha=135^{\circ}$. Thus, with higher pump frequency $f_{2}$ and higher pump frequencies $f_{1}$, the maximum amplitude of the generated wave is found at an interaction angles of $\alpha=45^{\circ}$ (see Fig. 7f). For any pump frequency value $f_{1}$ the amplitude of the generated wave is extremely low at $f_{2}<1 \mathrm{MHz}$ (see Fig. 7a-f). In order to generate a high amplitude acoustic wave from the mixing of two incident waves, the frequency $f_{2}$ must be higher than $1 \mathrm{MHz}$. For the remainder of the experiments, $f_{1}$ is chosen to be $650 \mathrm{kHz}$, while $f_{2}$ is varied between $850 \mathrm{kHz}$ and $1.35 \mathrm{MHz}$. The calculated interaction depth is $2 \mathrm{~mm}$.

\subsection{Experimental results}

A typical recorded signal is shown in the time domain in Fig. 8a. This signal is from the experiment with $f_{2}=1100 \mathrm{kHz}$. Fig. $8 \mathrm{~b}$ depicts the corresponding frequency spectrum. In the frequency domain, four frequency components are shown. The frequency components expected are $f_{1}, f_{2}$ and $f_{g}$. Another frequency is found at $f_{2-2 H}$. The latter corresponds to the second harmonic of the second pump wave. No further harmonics are present in the frequency spectrum. The amplitude of the generated wave can be extracted in the time domain with a band-pass filter. However, in this research, the extraction is done in the frequency domain. The maximum amplitude of the generated wave was extracted from the component $f_{g}$ as depicted in Fig. $8 \mathrm{~b}$.

There is a difference of approximately $30 \mathrm{~dB}$ between the amplitude of the second pump wave and that of the generated wave. A difference of approximately $5 \mathrm{~dB}$ was found between the first pump wave and the generated wave. The difference between the pump waves and the generated wave are due to the positioning of the receiver. A similar difference in amplitude is seen with the rest of the $f_{2}$ pump wave frequencies.

The amplitudes of the measured signals as a function of $f_{2}$ are shown in Fig. 9a and b together with the solution of Eq. (15). Solving Eq. (15) for only one interaction angle $\alpha$ does not account for beam divergence nor for the beam width. In the experiments, however, there is beam divergence with a finite width, so many interactions happen simultaneously even at conditions where the theory predicts the contrary. Eq. (15) was solved for $\pm 3^{\circ}$ with respect to the interaction angle $\alpha=98^{\circ}$ 

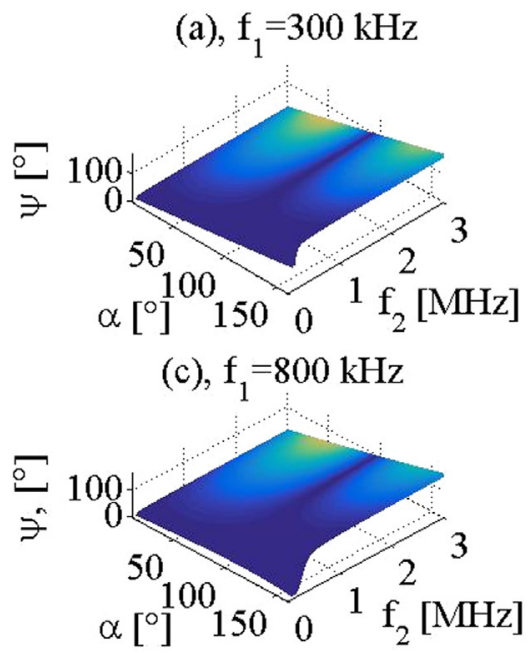

(e), $\mathrm{f}_{1}=1.3 \mathrm{MHz}$

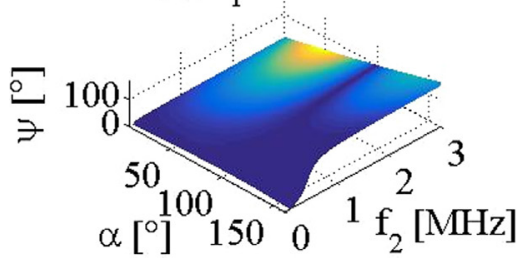

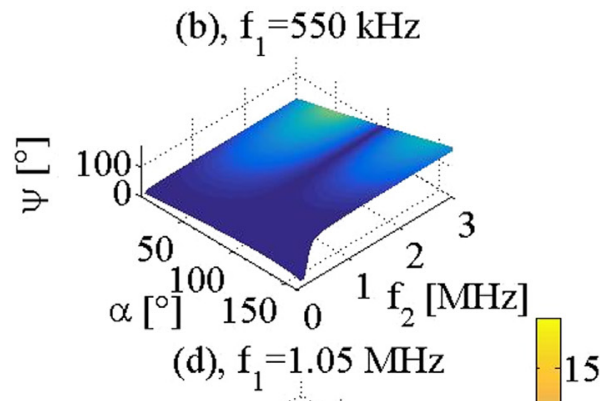
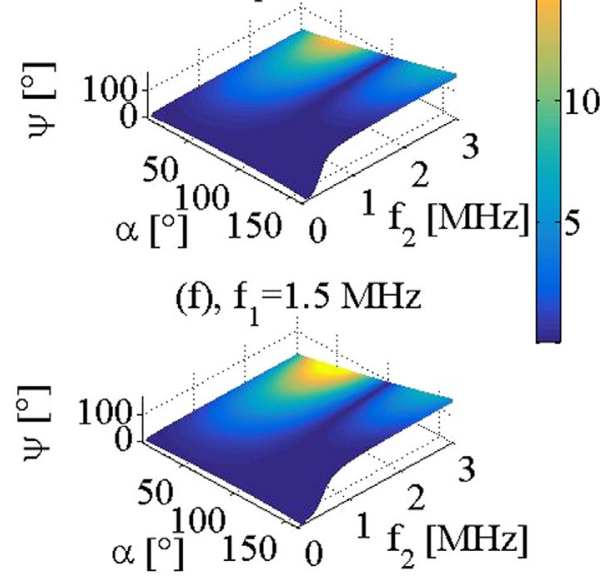

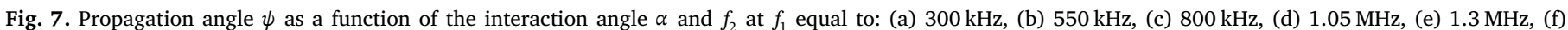

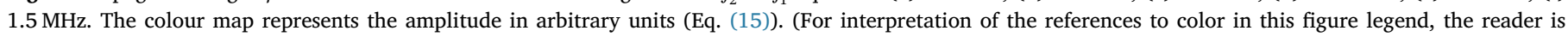
referred to the web version of this article.)

(see Fig. 9a). The solution of the 7 interaction angles $\left(98^{\circ}, \pm 1^{\circ}, \pm 2^{\circ}, \pm 3^{\circ}\right)$ was averaged and is depicted in Fig. 9b. The experiments correlate with the averaged analytical solution. A minimum value in both the experiments and analytic solution can be seen at a frequency $f_{2}=1.1 \mathrm{MHz}$. The experimental results show that the amplitude of the generated wave was never zero. No signal could be recorded after frequencies above $f_{2}=1.35 \mathrm{MHz}$, thus the analytical solution shown is also reduced to the range of $0.8-1.35 \mathrm{MHz}$.

\section{Conclusions}

In this research a solution of the scattering wave, generated from the mixing of two incident waves, is presented for the case where the angle of interaction is kept constant. The propagation direction of the generated wave is then steered by controlling the incident wave's excitation frequencies rather than the angle between the incident waves. This novel approach significantly reduces the complexity of a test set-up.

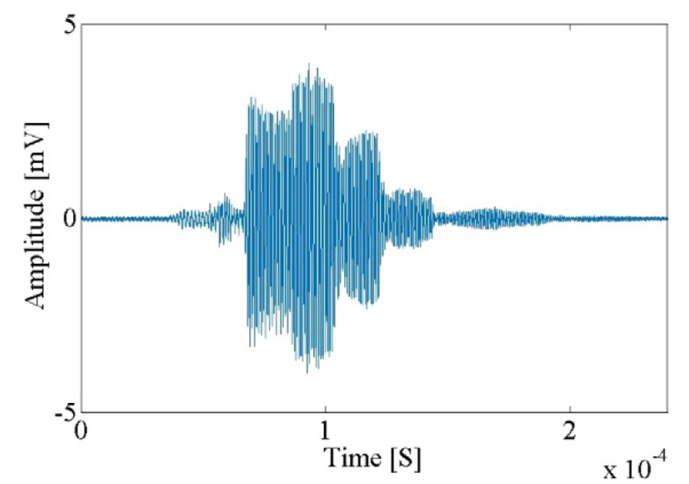

(a)
Rather than adjusting the positions of three sensors, only the receiver has to be adjusted. Another advantage of this solution is the possibility to adjust the excitation frequencies to obtain the maximum amplitude of the generated wave. This reduces the effects of positioning errors. It is of great advantage for NDE applications were several conditions restrict the testing configuration.

In previous research, the range of excitation frequencies are limited to the space and accessibility to the material. In this research, this is not a limitation anymore and a broader range of pump frequencies are available. The model used to demonstrate the proposed approach has been verified with some experiments, which confirms the potential of the method.

\section{CRediT authorship contribution statement}

Hector Hernandez Delgadillo: Conceptualization, Methodology, Software, Validation, Formal analysis, Investigation, Resources, Data

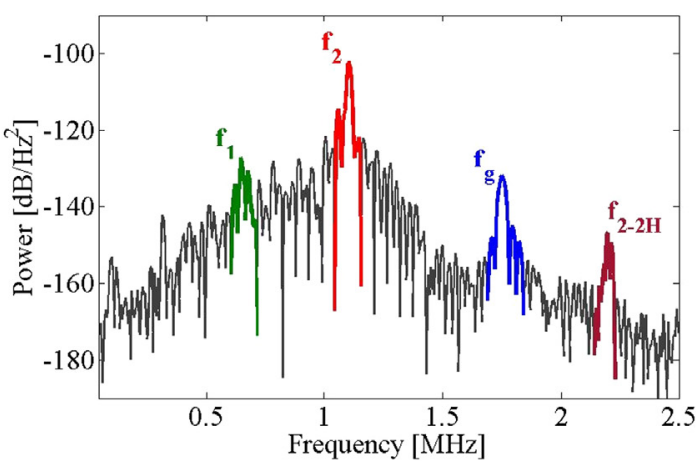

(b)

Fig. 8. (a) Time domain raw data from the experiments with $f_{2}(=1100 \mathrm{kHz})$; (b) the frequency spectrum of the respective signal $f_{g}(=1.75 \mathrm{MHz})$. 


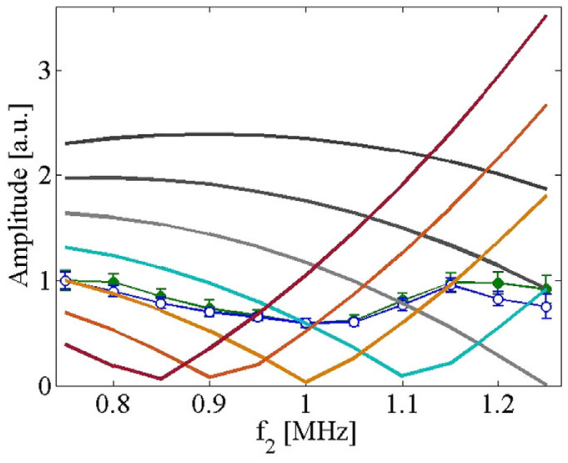

(a)

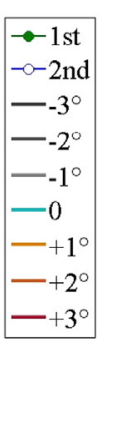

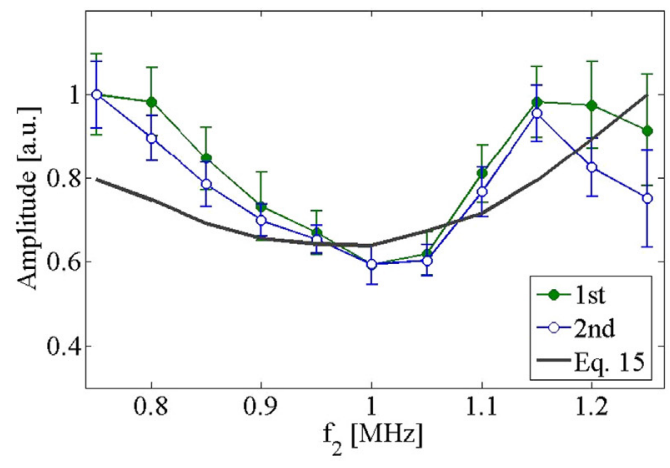

(b)

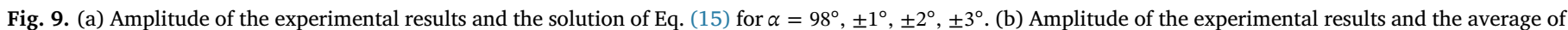
the solution of Eq. (15).

curation, Writing - original draft, Writing - review \& editing, Visualization, Project administration. Richard Loendersloot: Conceptualization, Formal analysis, Resources, Writing - review \& editing, Supervision, Project administration, Funding acquisition. Doekle Yntema: Conceptualization, Formal analysis, Resources, Writing - review \& editing, Supervision, Project administration, Funding acquisition. Tiedo Tinga: Conceptualization, Formal analysis, Resources, Writing - review \& editing, Supervision, Project administration, Funding acquisition. Remko Akkerman: Resources, Writing review \& editing, Supervision, Project administration, Funding acquisition.

\section{Acknowledgment}

This work was performed in the cooperation framework of Wetsus, European Centre of Excellence for Sustainable Water Technology (www.wetsus.eu). Wetsus is co-funded by the Dutch Ministry of Economic Affairs and Ministry of Infrastructure and Environment, the Province of Fryslân, and the Northern Netherlands Provinces. The authors like to thank the participants of the research theme "Smart Water Grids" for the fruitful discussions and their financial support. The authors thank to Hakan Kandemir for his help with the analytical solutions.

\section{References}

[1] S. Mezil, N. Chigarev, V. Tournat, V. Gusev, Evaluation of crack parameters by a nonlinear frequency-mixing laser ultrasonics method, Ultrasonics 69 (2016) 225-235, https://doi.org/10.1016/j.ultras.2016.04.005.

[2] H. Lv, J. Jiao, B. Wu, C. He, Evaluation of fatigue crack orientation using noncollinear shear wave mixing method, J. Nondestruct. Eval. 37 (4) (2018) 1-16, https://doi.org/10.1007/s10921-018-0523-z.

[3] J. Jiao, J. Sun, N. Li, G. Song, B. Wu, C. He, Micro-crack detection using a collinear wave mixing technique, NDT E Int. 62 (2014) 122-129, https://doi.org/10.1016/j. ndteint.2013.12.004.

[4] M. Sun, Y. Xiang, M. Deng, J. Xu, F.Z. Xuan, Scanning non-collinear wave mixing for nonlinear ultrasonic detection and localization of plasticity, NDT E Int. 2018
(93) (2017) 1-6, https://doi.org/10.1016/j.ndteint.2017.09.010.

[5] a. Demčenko, R. Akkerman, P.B. Nagy, R. Loendersloot, Non-collinear wave mixing for non-linear ultrasonic detection of physical ageing in PVC, NDT E Int. 49 (2012) 34-39, https://doi.org/10.1016/j.ndteint.2012.03.005.

[6] C. Pecorari, Modeling non-collinear mixing by distributions of clapping microcracks, Wave Motion 59 (2015) 69-80, https://doi.org/10.1016/j.wavemoti.2015. 08.001.

[7] G. Tang, M. Liu, L.J. Jacobs, J. Qu, Detecting localized plastic strain by a scanning collinear wave mixing method, J. Nondestruct. Eval. 33 (2) (2014) 196-204, https://doi.org/10.1007/s10921-014-0224-1.

[8] E. Escobar-Ruiz, A. Ruiz, W. Hassan, et al., Non-linear ultrasonic NDE of titanium diffusion bonds, J. Nondestruct. Eval. 33 (2) (2014) 187-195, https://doi.org/10. 1007/s10921-013-0217-5.

[9] Zhang Z. Nonlinear, Assessment of material and interface imperfections based on non-collinear shear, Wave Mix. (2016).

[10] Z. Zhang, P.B. Nagy, W. Hassan, Analytical and numerical modeling of non-collinear shear wave mixing at an imperfect interface, Ultrasonics 65 (2016) 165-176, https://doi.org/10.1016/j.ultras.2015.09.021.

[11] T. Ju, J.D. Achenbach, L.J. Jacobs, J. Qu, A non-collinear mixing technique to measure the acoustic nonlinearity parameter of an adhesive bond from one side of the sample, AIP Conf. Proc. 1806 (2017), https://doi.org/10.1063/1.4974552.

[12] A. Demčenko, L. Mainini, V.A. Korneev, A study of the noncollinear ultrasonicwave-mixing technique under imperfect resonance conditions, Ultrasonics 57 (2015) 179-189, https://doi.org/10.1016/j.ultras.2014.11.009.

[13] M. McGovern, H. Reis, Damage characterization in dimension limestone cladding using noncollinear ultrasonic wave mixing, Opt Eng. 55 (1) (2015) 011012, https:// doi.org/10.1117/1.OE.55.1.011012.

[14] S.K. Chakrapani, D.J. Barnard, Determination of acoustic nonlinearity parameter $(\beta)$ using nonlinear resonance ultrasound spectroscopy: theory and experiment, $\mathrm{J}$. Acoust. Soc. Am. 141 (2) (2017) 919-928, https://doi.org/10.1121/1.4976057.

[15] M. Liu, G. Tang, L.J. Jacobs, J. Qu, Measuring acoustic nonlinearity parameter using collinear wave mixing, J. Appl. Phys. 112 (2) (2012), https://doi.org/10.1063/1. 4739746.

[16] L.H. Taylor, F.R. Rollins, Ultrasonic study of three-phonon interactions. I. Theory, Phys Rev. 136 (3A) (1964) 136.A591, https://doi.org/10.1103/PhysRev.

[17] A.C. Holt, J. Ford, Theory of ultrasonic three-phonon interactions in single-crystal solids, J. Appl. Phys. 40 (1) (1969) 142-148, https://doi.org/10.1063/1.1657019.

[18] G.L. Jones, D.R. Kobett, Interaction of elastic waves in an isotropic solid, J. Acoust. Soc. Am. 35 (1) (1963) 5-10, https://doi.org/10.1121/1.1918405.

[19] Y. Hiki, K. Mukai, Ultrasonic three-phonon process in copper crystal, J. Phys. Soc. Jpn. 34 (2) (1973) 454-461, https://doi.org/10.1143/JPSJ.34.454.

[20] V.A. Korneev, A. Demčenko, Possible second-order nonlinear interactions of plane waves in an elastic solid, J. Acoust. Soc. Am. 135 (2) (2014) 591-598, https://doi org/10.1121/1.4861241. 